Voix et Images

\title{
Les énigmes des Fous de Bassan : féminisme, narration et clôture
}

\section{Marylin Randall}

Volume 15, numéro 1 (43), automne 1989

Jacques Poulin

URI : https://id.erudit.org/iderudit/200817ar

DOI : https://doi.org/10.7202/200817ar

Aller au sommaire du numéro

Éditeur(s)

Université du Québec à Montréal

ISSN

0318-9201 (imprimé)

1705-933X (numérique)

Découvrir la revue

Citer cet article

Randall, M. (1989). Les énigmes des Fous de Bassan : féminisme, narration et clôture. Voix et Images, 15(1), 66-82. https://doi.org/10.7202/200817ar d'utilisation que vous pouvez consulter en ligne.

https://apropos.erudit.org/fr/usagers/politique-dutilisation/ 


\section{Les énigmes des Fous de Bassan: féminisme, narration et clôture}

\section{par Marilyn Randall, University of Western Ontario}

La diversité des lectures suscitées par les Fous de Bassan d'Anne Hébert n'arrive pas à expliquer la fascination qu'exerce ce roman énigmatique, le sentiment ne de pas avoir sondé à fond le mystère de Griffin Creek. Roman policier par excellence, il se termine sur une confession du criminel qui laisse pourtant perplexe: c'est comme si l'accumulation d'indices menant à la résolution du meurtre et à la culpabilité finale de Stevens Brown s'accompagnait, à l'insu du lecteur, d'un ensemble de contre-indices travaillant sournoisement à miner sa certitude. Et l'incertitude se concrétise jusqu'à s'inscrire dans la phrase finale du texte, où Stevens admet que ses aveux ont été rejetés par la cour et considérés comme extorqués et non conformes à la loi ${ }^{1}$. Seul, à notre connaissance, parmi les commentateurs du roman, Jean-Louis Backès est sensible à cette ambiguité qui pose un ensemble de problèmes d'interprétation qui, comme nous le montrerons, ne peuvent être résolus de façon simple et univoque:

Un doute plane en effet sur ce récit trop bien construit. Il plane sur sa véracité, peut-être parce quelques lecteurs détestent les solutions trop nettes comme ils abhorrent le happy end. 2

$\mathrm{Si}$ les critiques ont trouvé dans ce texte-énigme une source de lectures riches et multiples (lecture postmoderne ${ }^{3}$, lecture féministe ${ }^{4}$, lecture mytho-biblique 5 ,

1 Anne Hébert, les Fous de Bassan, Paris, Seuil, 1982, p. 249. Toutes les citations renvoient à cette édition.

2 Jean-Louis Backès, «Le retour des morts dans l'œuvre d'Anne Hébert», Esprit créateur, vol. XXIII, no 3, automne 1983, p. 56.

3 Janet Paterson, «L'envolée de l'écriture: les Fous de Bassan d'Anne Hébert», Voix \& images, vol. IX, no 3, printemps 1984, p. 143-151.

4 Neil Bishop, «Énergie textuelle et production de sens: images de l'énergie dans les Fous de Bassan d'Anne Hébert», University of Toronto Quarterly, vol. LIV, no 2, hiver 1984-1985, p. 178-199; Karen Gould, «Absence and Meaning in Anne Hébert's les Fous de Bassan», French Review, vol. LIX, n०6, mai 1986, p. 921 930; Kathryn Slott, «Submersion and Resurgence of the Female Other in Anne Hébert's les Fous de Bassan», Québec Studies, 4, 1986, p. 158-169; K. Sloth, «Repression, Obsession, and Re-emergence in Hébert's les Fous de Bassan», American Review of Canadian Studies, vol. XVII, n 3, 1987, p. 297-307.

5 Antoine Sirois, «Bible, mythes et Fous de Bassan d'Anne Hébert», Canadian Literature, 104, printemps 1985, p. 178-182; Yvette Francoli, «Griffin Creek: refuge des Fous de Bassan et des bessons fous», Études littéraires, vol. XVII, no 1 , avril 1984, p. 131-142; Ruth Mésavage, «L'herméneutique de l'écriture: les Fous de Bassan d'Anne Hébert», Québec Studies, no 5, 1987, p. 111-123. 
lecture narrative ${ }^{6}$ ), certaines de leurs conclusions s'avèrent, en effet, assez homogènes et se rejoignent sur deux points fondamentaux. Premièrement, les six livres qui composent les Fous de Bassan représenteraient cinq voix narratives distinctes, exprimant le point de vue du personnage incarné par le «je» autodiégétique; deuxièmement, la confession par laquelle le roman se termine fournirait une résolution véridique et non problématique au mystère. Et, selon une troisième tendance, celle des lectures féministes, le texte revendiquerait une position féministe en articulant une idéologie de la libération et de l'épanouissement des femmes ${ }^{7}$. Ces trois affirmations sont nécessairement liées, car c'est grâce au présupposé selon lequel chaque récit livrerait la vérité intérieure de son personnage que l'on arrive à justifier la revendication féministe: par les récits de Nora et d'Olivia nous seraient révélées leurs propres voix, afin qu' elles reprennent le pouvoir de l' expression de soi, et pour parler librement de leur désir ${ }^{8}$. Le même présupposé sous-tend également l'authenticité de la confession de Stevens, qui semble combler le vide laissé par le récit du meurtre dans les narrations précédentes.

\section{Féminisme}

L'argument féministe semble avoir pour justification principale la force étonnante du désir obsessionnel de deux filles innocentes pour un homme misogyne. Nora, par exemple, ne fait nul mystère de son désir pour Stevens - je suis creuse et humide. En attente, et Olivia, pour sa part, reconnaît et parfois semble éprouver encore ce désir au-delà de la mort même (p. 221-223) ${ }^{9}$. La victoire de la femme serait le fait de «survivre», en quelque sorte, à son meurtre par Stevens qui ne réussit pas à abolir le désir de la femme en abolissant son corps: Son désir est encore plus avide et plus présent après sa mort que dans sa vie. ${ }^{10}$ La lecture féministe semble donc vouloir compenser le sort des femmes (stérilité, suicide, subjugation sexuelle, viol, meurtre) par la force des voix féminines qui sont bien, aussi, des voix féministes. Certes, il s'agit d' un féminisme peu conscient de lui-même, mais non moins réel pour autant ${ }^{11}$. Cependant, l'évidence textuelle réduit ce féminisme inconscient à peu de chose: aux voix des femmesancêtres soufflant dans le vent mais impuissantes à sauver Olivia et Nora du sort que leur destine, semble-t-il, leur propre désir; à un rêve de jeune fille d'un prince charmant qui soit beau (Stevens), qui ne soit ni vieux, ni laid, ni fou.

Quant à la position de supériorité morale des femmes ${ }^{12}$, on peut facilement admettre qu'elles ne sont pas criminelles au sens de Stevens. Cependant, la

6 Neil Bishop, «Distance, point de vue, voix et idéologie dans les Fous de Bassan d'Anne Hébert», Voix \& images, vol. IX, n² 2, hiver 1984, p. 113-129.

7 Neil Bishop, ibid., p. 129. Pour une exception à cette lecture, voir Suzanne Lamy, «Un livre de l'irresponsabilite», Spirale, n²9, novembre 1982, p. 3, 2.

8 Kathryn Slott, «Repression...», loc. cit., p. 298.

9 Neil Bishop, «Distance...", loc. cit., p. 123.

10 Karen Gould, loc. cit., p. 938.

11 Neil Bishop, loc. cit., p. 124.

12 Kathryn Sloth, «Submergence...», loc. cit., p. 158. 
froideur maternelle de Bea et de Felicity, la vengeance sexuelle, la jalousie et la provocation exercées par Nora se semblent guère correspondre ni à la vertu ni à la moralité. Qui plus est, la question des «voix des femmes» pose de nombreux problèmes pour une lecture qui défend la notion d'une émergence du pouvoir féminin. Cet argument aboutit à une position narrative quelque peu paradoxale, sinon indéfendable, qui prétend racheter la soumission des femmes à l'autorité patriarcale par le silence et la contrainte même des voix féminines ${ }^{13}$.

Les divers points de vue fournis par les multiples récits ont suscité un vif intérêt pour des questions narratives dans l'analyse du roman. Mais le problème qui surgit à l'égard de la présumée pluralité des voix narratives est plutôt leur étonnante homogénéité. De livre en livre, de narrateur à narratrice, les mêmes images, les mêmes discours, les mêmes champs sémantiques obsessionnels reviennent inlassablement. Car le texte ne se répète pas uniquement par le truchement du retour anaphorique aux scènes et aux événements de l'été $1936^{14}$. Que ce soit au niveau d'un réseau sémantique d'images animalesques, dominé par les oiseaux et les poissons, ou bien au niveau des refrains répétés par chacun des personnages, tel Non, non, ce n'est pas Stevens et Cette fille est folle (Nicolas, p. 37, Stevens, p. 244), l'aspect obsessionnel du texte est évident ${ }^{15}$.

Les réseaux obsessionnels reliant les univers conceptuels des personnages révèlent chez eux une psychologie commune. Par l'entremise de la citation biblique: Et le Verbe s'est fait chair et Il a habité parmi nous (p. 118), par exemple, Nicolas, Stevens et Nora s'attribuent une identité semblable. Nicolas déclare avoir été le Verbe de Griffin Creek (p. 20), tandis que Stevens, dans un passage qui paraît répondre directement à celui de Nicolas, déclare: Si quelqu' un ressemble au Christ dans ce village, c'est bien moi, Stevens Brown (p. 89). Et Nora utilise la même image, déclarant: Le Verbe en moi est sans parole prononcée, ou écrite, réduite à un murmure secret dans mes veines (p. 118) (affirmation qui trouble quelque peu aussi son statut de narratrice - nous y reviendrons).

Par le biais des obsessions fixées sur la mère, il est évident que Stevens et Nicolas voient le monde et leur expérience de façon identique. Obsédés tous deux par Felicity, la grand-mère du premier et la mère du deuxième, ils sont d'accord pour sentir qu'elle les a privés d'amour et qu'elle aime seulement ses filles et petites-filles. Et, en fait, d'après les témoignages de leurs fils, petit-fils et mari, Felicity et Bea, ainsi qu'Irène, l'anti-mère, manifestent une froideur et sont atteintes d'une stérilité qui remet radicalement en question l'affirmation d'une critique voulant que les hommes se séparent de l'amour et de la tendresse ${ }^{16}$. Selon la psychologie exposée dans les récits de Nicolas et de Stevens, la cause

13 Ibid., p. 167-168.

14 Janet Paterson a étudié ces retours sémantiques à l'égard de la structure anaphorique dans une communication inédite donnée à Chicago, au congrès de la Modern Language Association en décembre 1985.

15 Kathryn Slott analyse la psychologie des obsessions dans «Repression, Obsession and Re-emergence in Anne Hébert's les Fous de Bassan», loc. cit.

16 Ibid., p. 160. 
de leur misogynie reposerait sur une enfance hantée par le désir insatisfait de l'amour maternel et par un refus de tendresse de la part des femmes.

Au sujet des parents de Stevens, les deux hommes partagent aussi la même fantaisie enfantine du conte de fée où les parents rêvent de se débarrasser de leurs enfants:

Leurs père et mère ayant désiré très tôt les [les sours de Stevens] perdre en forêt n'ont pas fait de manières pour me les céder, à l'âge de treize ans. (Nicolas, p. 19)

John et Bea Brown ayant mis au monde Stevens, Perceval et les jumelles, s'en sont débarrassés, au cours d' un seul été. Réalisation d'un vieux rêve enfin justifié. Ne plus avoir d'enfant du tout. (Nicolas, p. 21)

Il est question d' enfants qui ne doivent pas naître et d' enfants déjà nés $q u$ 'il faut perdre en forêt, avant qu'ils ne soient trop grands. Je préviendrai Perceval et les jumelles. Je les emmènerai avec moi [...].

(Stevens, p. 85)

On aurait vite fait de régler la question de la répétition en mettant au jour une auteure implicite, source et origine de «l'univers hébertien» où l'on retrouve, en fait, le même procédé de répétition et une certaine thématique obsessionnelle. Mais les répétitions qui traversent les discours des narrateurs multiples et distincts ne se présentent pas uniquement comme un effet du style hébertien: elles génèrent par surcroît une ambiguîté irréductible à l'égard de l'interprétation qui dépend de l'identité et de l'autorité de ces voix. En fait, plutôt que de présenter une epluralité» de points de vue sur un même événement ${ }^{17}$, les récits successifs révèlent une surprenante unité de vision. Prenons, par exemple, la question du désir.

Stevens, misogyne, machiste, sadique et mégalomane, attribue à certains personnages féminins (Olivia, Nora et Maureen) un désir passionné à son endroit. Selon Neil Bishop, la vision de Stevens serait caractérisée par un degré $d^{\prime}$ '«omniscience» 18 qui s'accorderait parfaitement avec son égoïsme et ses illusions de grandeur, car ses perceptions se trouvent apparemment confirmées par les récits de Nora et d'Olivia, dont le désir pour Stevens s'avère aussi obsessionnel qu'il pourrait l'imaginer.

L'homogénéité profonde qui sous-tend certaines des scènes récurrentes ne contribue qu'à problématiser le présupposé de sources narratives différentes. Dans la scène de la «chasse», par exemple, où Nora «traque» Stevens dans le bois, Bishop voit un exemple de la différence des points de vue entre les deux narrateurs parce que, contrairement à l'attitude de domination de Stevens, Nora exprime le désir d'égalité entre les deux passions qui s'affrontent. Or, notre lecture des deux scènes souligne plutôt leur conformité. Dans la lettre de Stevens, on lit:

17 Neil Bishop, «Distance...», loc. cit., p. 120.

18 Ibid., p. 120-121. 
Elle s'arrête et me regarde avec des yeux de lièvre, surpris par le chasseur [...] Je crois aussi qu' elle me supplie de l'embrasser. Je l'embrasse aussitôt, sur le bout des lèvres, sur les deux joues [...].

- Il ne faut pas faire ce que tu pourrais regretter. (p. 90-91)

Or, la «preuve» qu'il voit juste serait fournie dans le passage où Nora raconte la même scène:

$C^{\prime}$ est lui le chasseur et moi je tremble et je supplie quoique j' enrage d'être aussi tremblante et suppliante en silence devant lui [...].

- Il ne faut pas faire ce que tu pourrais regretter. [...] Il a refusé de m'embrasser comme un homme embrasse une femme et moi je l'attendais, depuis le matin, m'attachant à ses pas pareille à un chien de chasse qui suit une piste. (p. 127)

Ainsi, l'attitude de supplication et le rôle d'animal traqué dont Stevens investit Nora seraient pleinement assumés par elle. Mais l'apparente omniscience de Stevens n'est que son interprétation subjective, et Stevens luimême ne prétend pas autre chose, dans la mesure où ses remarques sont souvent préfacées d'une modalisation de doute ${ }^{19}$. Si Stevens possède une certaine omniscience, celle-ci est attestée par les témoignages des autres, qui n'ont pour fonction que de corroborer sa vision de Griffin Creek.

Les hommes, c'est-à-dire Stevens et Nicolas, sont d'accord pour culpabiliser les femmes, pour trouver qu'elles sont à l'origine du malheur, du péché, de la faute originelle. Or, voilà que la comparaison des récits des filles avec la vision des hommes fournit encore une confirmation de cette dernière, situation dont la lecture féministe ne rend pas compte.

Il y a trop de femmes dans ce village, trop de femmes en chaleur et d'enfants perverses qui s'attachent à mes pas (p. 80), dira Stevens. Quant à Nicolas, tourmenté par la beauté adolescente de Nora, il cède à la tentation et commet de la cochonnerie avec elle dans la cabane à bateaux. Or, cette cochonnerie s'avère, finalement, une manœuvre orchestrée par Nora dans le seul but de se venger de Stevens qui a repoussé ses avances sexuelles. La signification de la scène racontée dans le livre de Nora ne peut laisser insinuer aucun doute: c'est Nora, femme rejetée par l'objet de son désir, qui joue pleinement le rôle que Stevens et Nicolas lui attribuent:

Je crois que je commence à attendre l'événement qui me vengera de Stevens. [...] Je me promène sur la grève, près de la cabane à bateaux. I'attends que l'événement se produise. (p. 128)

19 Au passage cité, s'ajoutent d'autres exemples de cette modalité de subjectivité et de doute, clairement exprimée par Stevens: Je crois que cette femme [Maureen] est heureuse de nourrir un homme [...] (p. 66); On dirait que cette femme [...] se trouve rassasiée [...] (p. 67); Certaine phrase a-t-elle été vraiment dite par Olivia $[$...], ou l'ai-je imaginée [...]? (p. 79). 
Moi aussi, j'ai été cochonne avec le pasteur, dans la cabane à bateaux. Pour me venger de Stevens. (p. 130-131)

Et le pasteur, pour sa part, vieux salaud qu'il est, ne semble pas avoir rempli la fonction de séducteur agressif qu'on lui assigne. Toujours hanté par son péché après quarante-six ans, il réfléchit:

Je ne saurai sans doute jamais d' où lui venait sa fureur, ce matin-là, en ai profité comme quelqu' un qui ramasse les miettes sous la table. (p. 45)

Le témoignage de Nora, Ève nouvelle, semble confirmer que c'est par elle que la faute est entrée à Griffin Creek. La provocation qu'elle exerce afin d'être séduite présente un problème évident pour une lecture qui valorise l'innocence des filles et la supériorité morale des femmes. Pour s'en sortir, les critiques féministes sont amenées à parler de la contamination du désir féminin par la lubricité des hommes ${ }^{20}$ et à interpréter l'usage par Nora du mot cochonnerie comme symptôme du processus par lequel l'homme culpabilise le désir féminin 21 .

Il y a pourtant très peu d'indications, si l'on exclut le mot cochonnerie, prouvant que Nora ait «intériorisé» cette culpabilité. Son emploi du mot pour décrire la scène avec Nicolas suggère bien sûr qu'elle reconnaît dans son expérience un acte sexuel pervers, qu'elle distancie d'un autre type de rapport sexuel qui répondrait à son rêve idéal. Elle sait qu'elle a été «cochonne» avec le pasteur (Mon Dieu quel péché est-ce là! [p. 131]) parce que son acte de manipulation est motivé par la vengeance et la jalousie. Sa réaction à sa «séduction» confirme que cette cochonnerie est un acte lucide qui ne l'empêche ni de se croire innocente ni de valoriser l'amour sexuel. C'est après l'aveu de sa cochonnerie qu'elle déclare:

Mon Dieu donnez-moi bien vite un garçon de mon âge qui ne soit pas marié ni pasteur. Pour le fun de tout mon corps, né pour cela, de la tête aux pieds, pour l'amour de toute mon âme, née pour cela, dans sa sauvage innocence. (p. 131)

C'est également à l'égard du rôle des forces naturelles associées à l'univers des femmes, où l'on retrouve une certaine univocité dans le texte, que le discours féministe exprime une ambiguîté assez troublante. Il n'y aurait aucun doute que le pouvoir des femmes se manifeste à travers leur relation à la mer et au vent, dont la puissance, liée aux origines créatrices du monde, envahit la vie entière de Griffin Creek. C'est surtout le retour d'Olivia comme revenante de la haute mer, emportée par le vent, qui établit la continuité de la force des femmes au-delà de la mort, et au-delà de la sphère des hommes. Les forces puissantes et éventuellement destructrices de la mer, du vent et de l'orage sont indissociables d'un pouvoir qui semble être l'apanage des femmes.

20 Kathryn Slott, «Submergence... », loc. cit., p. 164.

21 Neil Bishop, «Distance...», loc. cit., p. 125. 
Or, une contradiction s'insinue dans cette interprétation du moment où le vent participe au réseau des forces négatives, destructrices, qui rendent fou et qui provoquent, finalement, le meurtre des deux filles et la fin du monde à Griffin Creek. Dès que le vent s'avère agressif et maléfique, il est assimilé à la passion sexuelle, destructrice des hommes, de sorte qu'il devient une métaphore du désir masculin destructeur 22. Mais un curieux syllogisme vient infirmer cette interprétation. Le vent est clairement présenté comme une force féminine, originaire de la mer, et porteuse des voix des femmes-ancêtres, des femmes mortes, dont Olivia. Et le vent rend fou. Mais le vent n'est pas la seule source de folie à Griffin Creek. N'est-ce pas la simple présence des femmes qui, non moins que le vent, engendre la folie chez les hommes? Si l'origine de la rage et de la folie de Stevens, de Perceval et de Nicolas est le vent, l'équivalence établie entre le vent et les femmes impose la conclusion que ce sont également les femmes qui suscitent la rage. Sur la plage, la nuit du 31 août 1936, Stevens était emporté par un vent fou qui n'existait pas, par la passion que déclenche la présence des femmes. Au lieu de représenter ce désir masculin destructeur, le vent, c'est à-dire les femmes, le provoque. Dans toute cette histoire, il faut tenir compte du vent, de la force enrageante» des femmes.

Curieux livre féministe, donc, que celui-ci où les femmes, en plus d'être soumises jusqu'à leur mort, se conforment, en fait, selon leur propre témoignage, à chaque fantaisie que les hommes les plus misogynes peuvent entretenir à leur sujet.

La contradiction dans l'argument féministe apparaît clairement chez Kathryn Slott qui, tout en affirmant la liberté naissante de la parole libératrice des femmes, doit admettre qu'Olivia est morte avant même de pouvoir découvrir sa voix véritable 23 . De cette contradiction - celle impliquée par le pouvoir d'une voix muette - nous ne sortirons pas, car Nora elle-même affirme son mutisme dans une phrase qui doit étonner, du fait qu'elle se retrouve inscrite dans un «livre» dont elle serait, sinon la scriptrice, du moins la narratrice:

Et moi aussi, Nora Atkins, je me suis faite chair et j' habite parmi eux, mes frères et mes cousins de Griffin Creek. Le Verbe en moi est sans parole prononcée, ou écrite, réduit à un murmure secret dans mes veines. (p. 118)

La conclusion des lectures féministes reste donc tiraillée entre la domination absolue des femmes par les hommes et la nécessité, implicite dans leur programme de lecture, de revendiquer pour les femmes un pouvoir, ne serait-ce que virtuel. Ce pouvoir est réalisé, en l'occurrence, dans un irréel mythologisant:

D'une part, Hébert a démontré la privation du pouvoir des voix des femmes par le patriarcat; de l'autre part, elle a réinvesti ce pouvoir

22 Neil Bishop, «Énergie...», loc. cit., p. 189.

23 Kathryn Slott, «Repression...», loc. cit., p. 298. 
d'une force et d'une vision renouvelées, en situant l'énergie sousjacente à son roman dans l'image archétypale de la mer et dans une généalogie mythique du matriarcat. ${ }^{24}$

Il est vrai que l'énergie passionnée des femmes est une force qui s'affirme au-delà de la mort et qui s'oppose aux pulsions masculines de domination. Mais il est difficile de voir dans ce recours au symbolisme archétypal, lui-même originaire d'une mythologie patriarcale, un «renouvellement» d'une force et d'une vision féminines. Définitivement domptée dans la vie, cette voix féminine revendicatrice de sa liberté et de son désir, n'a elle-même qu'un pouvoir négatif dont elle est finalement prisonnière éternelle. Incapable de quitter la scène de son désir et de son meurtre, la femme est impuissante dans l'acte et dans la parole, dans la mort comme dans la vie.

Les Fous de Bassan mettrait donc plutôt en scène la privation totale de la parole - prononcée ou écrite - des femmes. Le Verbe dans les Fous de Bassan est irréfutablement masculin: l'Histoire, celle du monde à Griffin Creek, ancrée dans la Parole de Dieu, en surgit. L'homme est le Verbe fait chair et énoncé, tandis que chez la femme, le Verbe est réduit à un murmure secret dans [ses] veines - la femme n'y est que corps désirant et désiré, qui participe pleinement à une mythologie du matriarcat où se réinscrit la peur archaïque chez l'homme des forces naturelles (féminines) déchaînées, dont la constellation comprend la mer, la folie et la marâtre.

Les aspects ambigus du mythe cosmogonique sont soulignés surtout par la parenté entre le couple Nora-Olivia et la figure cabalistique de Lilith. Tout comme Nora, cette première Ėve fut créée du limon de la terre, comme Adam, et non sortie d'entre les côtes sèches d'Adam, première comme Adam [...] (p. 116). Son insoumission à Adam et à Dieu lui valut l'expulsion de la terre. Désormais associée au vent, surtout à l'orage, et à la mer qui deviendra son domicile, elle ne cesse d'être tourmentée par une perversion du désir, qui l'éloigne de la participation aux normes ${ }^{25}$. Ennemie d'Ève, des enfants et de la famille, la symbolique de revendication féministe est teintée d'une tradition négative - dans ses rôles de tentatrice vengeresse de l'homme et de mère terrible, elle incarne les forces psychologiques du mal féminin ${ }^{26}$.

C'est en fait l'impuissance, voire l'absence, du Verbe féminin dans cette mise en scène de paroles qui constitue l'élément féministe le plus radical du texte et qui entraîne la condamnation du monde patriarcal représenté dans la fiction. Et l'insistance de Nora sur le fait que le Verbe en elle est sans parole prononcée, ou écrite, sert à souligner l'autre versant de la question des voix narratives, celle qui se pose par la présence évidente d'une voix narrative organisatrice, de l'ordre d'un scripteur implicite. En effet, il est évident qu'avant

24 Kathryn Slott, «Subversion...», loc. cit., p. 168.

25 Jean Chevalier, Dictionnaire des symboles, Paris, Robert Laffont, 1969, p. 461.

26 Ad de Vries, Dictionary of Symboles and Imagery, Amsterdam, North Holland Pub. Co., 1974, p. 297-298. 
d'être en présence d'une parole, on est en présence d'une écriture. Livre de livres, où l'aspect livresque est souligné par l'analogie biblique et par les multiples pages de titre, les Fous de Bassan posent l'épineuse question de savoir qui écrit?

\section{Narration}

La présence de narrateurs multiples, chacun mettant en scène une certaine "vérité», semble favoriser l'indécidabilité et l'ouverture du récit vers un domaine de l'expérience humaine où la notion de «vérité» perd sa transcendance et s'installe irrémédiablement chez le sujet connaissant. Radicalement antipathique aux présupposés du roman policier traditionnel, le maintien de paradoxes irréductibles par le biais des points de vue multiples est une stratégie pourtant commune au texte moderne (on cite à ce propos l'affinité entre Hébert et Faulkner ${ }^{27}$ ): la Vérité ressortirait de l'accumulation de vérités partielles, subjectives, relatives. Or, cette convention moderne repose sur le présupposé de la transparence du sujet du discours à lui-même, transparence que la mise en discours du sujet par lui-même ne peut, paraît-il, troubler. Pourtant, l'utilisation de la technique des voix narratives multiples trahit une certaine méfiance quant à ce présupposé de transparence, si bien que dans certains cas exemplaires de la forme, chez Nathalie Sarraute et Virginia Woolf par exemple, le garant de cette fidélité transparente entre le sujet et son discours passe par la création d'un narrateur anonyme dont la seule fonction est de foumir une traduction linguistique des états pré-linguistiques du personnage. Dans ces discours, la voix narrative est la condition de la mise en discours du sujet présent à lui-même, mais qui ne se connaît pas en langage.

Or, en dépit de l'évidence narrative qui devrait susciter une méfiance vigoureuse face à la notion de vérité discursive, les anciens présupposés de la transparence semblent gouverner la lecture critique de la narration des Fous de Bassan. Dans ce texte, la voix narrative personnelle prétend fonctionner comme une fenêtre donnant sur le vrai monde de la psychologie profonde du sujet. Ici, par le truchement apparent de monologues intérieurs, de «livres», de lettres, les personnages se racontent dans des discours qui semblent, selon la critique hébertienne, participer à cette transparence parfaite mais illusoire grâce à laquelle le sujet parlant et le sujet parlé coïncident. Autrement dit, mais contre toute attente, le discours des je-narrateurs serait vrai en vertu même de sa subjectivité.

De ces contradictions narratives, on ne sortira pas sans présupposer une voix narrative organisatrice, dont la nécessité s'avère si puissante que, faute d'autre candidat, on projette la présence dans le texte de l'auteure elle-même. La signature hébertienne, qui s'inscrirait dans son récit par le biais d'obsessions familières à ceux et celles qui savent lire, est implicitement ou explicitement évoquée par ses lecteurs qui se plaisent à exposer l'identité d'un univers cohérent. Cette conclusion résulte de la question qui raconte le récit?, question transformée, sous

27 Ronald Ewing, «The English World of Griffin Creek», Canadian Literature, no 105 , été 1985 , p. 100-110. 
la pression du poids livresque du texte, en qui écrit le texte? La question s'impose inéluctablement et demande une réponse qui sauvera la vraisemblance ténue d'un récit «raconté» par une fille morte. Pourtant, répondre au paradoxe que pose la narration-écriture de ses discours indicibles en proposant Anne Hébert comme leur «organisatrice» me déçoit finalement, non pas uniquement à cause de mes propres présupposés méthodologiques, mais dans la mesure où cette réponse enlève une part du mystère et de la signification de cette prise de parole par des muets et des morts. Si le roman s'apparente au roman policier, c'est par l'énigme de sa composition: le vrai mystère à résoudre est: qui a écrit les Fous de Bassan?

La demière lettre de Stevens Brown, qui introduit une distorsion inattendue dans le récit, provoque une lecture rétrospective des livres précédents et permet de cristalliser leurs ambiguîtés déjà latentes. C'est dans cette lettre finale que la cohérence précaire du récit éclate sous la pression des contradictions et des énigmes textuelles accumulées. Au lieu d'en fournir la résolution, cette lettre installe plutôt l'incertitude quant à la vérité de toute finalité univoque.

Brisant l'espace romanesque pour nous situer dans un "présent réel» - Montréal, automne 1982 - la dernière lettre de Stevens à son ancien et toujours absent correspondant présente quelques anomalies, dont l'évocation de la guerre qui vient déranger une succession d'événements dans leur causalité linéaire. Sorti indemne de la guerre, Stevens n'est pas sans avoir été atteint dans sa santé mentale: il est, parait-il, fou - pourtant ne l'était-il pas déjà avant la guerre, comme il l'affirme dans une lettre de l'été 1936: semblable à un fou que je suis (p. 102). Ou bien la folie ne serait en fin de compte qu'un résultat de la guerre: C'est la guerre mon vieux, rien d'autre que les séquelles de la guerre, te dis-je, Griffin Creek n'y est pour rien [...] (p. 232). Quelle que soit la réponse à cette question, l'obsession du passé et la hantise de la culpabilité incitent Stevens à finalement tout confesser, à dire la vérité, Old Mic, toute la vérité, rien que la vérité (p. 237) - mais ne l'avait-il pas déjà avouée, cette vérité, dans la confession qu'on a jugée lui avoir été extorquée? En fait, les mystères s'accumulent dans cette demière lettre qui est bourrée d'indications obscures et d'apparitions brèves (p. 234) et qui finit par remettre en question tout ce qui précède, jusqu'à menacer la véracité de la confession même qu'elle constitue.

Enfin, l'accumulation d'énigmes atteint son comble quand ce narrateur détraqué et délirant annonce: Dans toute cette histoire, il faut tenir compte du vent, je l'ai déjà dit (p. 246. C'est nous qui soulignons). Mais ici, Stevens se trompe ou bien il ment. Cette phrase, il ne l'a jamais prononcée, et pourtant on la retrouve intégralement "sous la plume» d'un autre "narrateur», Nicolas Jones. Ou bien, dans le contexte de sa confession finale, Stevens dit vraiment la vérité, toute la vérité et rien que la vérité. Cette phrase, s'il l'a déjà dite, c'est à travers un autre personnage, et les Fous de Bassan seraient, par conséquent, le livre de Stevens Brown ${ }^{28}$. Cette hypothèse, qui reste invérifiable, permet de

28 Cette lecture est celle proposée par le film tiré du roman, où l'histoire se présente comme les souvenirs narrés par le vieux Stevens. Il n'est pas sans intérêt pour des questions théoriques d'intentionnalité et d'interprétation de savoir que cette lecture, 
donner un sens à des contradictions et à des énigmes qui, autrement, resteraient insolubles. Et la première d'entre elles est cette faille dans la narration de Stevens lui-même, lapsus dont il faut tenir compte et qu'il faut intégrer dans l'économie signifiante d'un texte qui refuse les solutions trop nettes tout en proposant la vérité d'une «confession» finale. Il s'agit donc d'examiner le statut de cette confession.

Évadé de 1'Institut, Stevens loue une chambre, s'équipe d'une quantité importante d'amphétamines et de barbituriques, et achète un cahier dans le seul but de raconter toute la vérité. Or, cette dernière lettre à Michael Hotchkiss ne répondrait pourtant pas à l'envergure du projet pour lequel Stevens se prépare. Ce projet, en effet, est plus grand. Apparentée à la galerie des ancêtres de Nicolas, la narration de Stevens propose d'évoquer, de recréer, voire de créer l'histoire et les motivations profondes des personnages impliqués dans le drame de l'été 1936: Le mieux ce serait de peindre comme on m'a appris à le faire à l' hôpital [...]. La vérité finirait bien par passer sur ma toile [...]. (p. 235)

Comme figure de l'artiste-scripteur, Stevens rejoindrait une lignée de scripteurs fictifs québécois dont André Belleau a déjà fait l'historique ${ }^{29}$. L'association entre l'artiste, l'écrivain et le meurtrier ne va pas non plus sans avoir ses antécédents dans la littérature québécoise, si l'on songe à Trou de mémoire d'Hubert Aquin dont le récit consiste en une confession par le narrateur du meurtre de son amante. Les voix narratives apparemment multiples intervenant dans chaque texte ne sauraient se maintenir dans leur intégrité, et se proposeraient finalement comme n'étant que la reprise continuelle d'une même obsession, d'une seule voix.

Dès ses premières lettres, comme le remarque Janet Paterson, Stevens s'érige en figure de créateur et d'écrivain:

«J' ai le pouvoir de faire exister mon grand-père, au bout de mon pied, ou de l'abandonner au silence d' un sommeil opaque.» (p. 64) Presque insignifiante dans le contexte de la deuxième lettre de Stevens, cette mise en abyme présage néanmoins la prise de pouvoir de Stevens par l'écriture dans sa dernière lettre: «Je dispose du jour et de la nuit à ma convenance... Faire venir le démon sur mon cahier, si la fantaisie $m^{\prime}$ en prend.» (p. 234) Ainsi d' une mise en abyme à l'autre, d'un récit à l'autre, le texte répercute des signes, des images et des fragments de l'activité créatrice. ${ }^{30}$

Or, cette prise de pouvoir par l'écriture, mise en abyme de l'activité créatrice, l'est au niveau même de la fiction et signale le pouvoir exercé par

qui enlève aux filles l'autorité de leurs voix, a suscité le désaveu d'Anne Hébert (Congrès du Conseil international d'études francophones, tenu à Montréal le 15 avril 1988).

29 André Belleau, le Romancier fictif, Québec, Presses de l'Université du Québec, 1980.

30 Janet Paterson, Anne Hébert: architexture romanesque, Ottawa, Éditions de l'Université d'Ottawa, 1985, p. 174. 
Stevens non seulement dans sa dernière lettre, mais sur l'ensemble du livre. Dès ses premières lettres, il affirme:

Je possède un pouvoir. Si tout à l' heure j'ai pu faire vivre mon grandpère, au bout de ma botte, je perçois maintenant un cri sourd dans la poitrine de Maureen. (p. 68)

Et ce pouvoir créateur est assumé explicitement par Stevens comme moyen de se dédoubler, de devenir un autre. En empruntant dans son livre les voix des protagonistes de l'été funeste, Stevens atteindrait son désir de raconter sa vie passée sans pour autant s'y identifier:

Être quelqu' un d'autre, quelle idée est-ce là qui me poursuit toujours. Organiser les souvenirs, disposer les images, me dédoubler franchement, tout en restant moi-même. Pouvoir témoigner de ma vie passée, sans danger, sans être obligé d'y entrer à nouveau et dire: Voilà c'est moi, Stevens Brown, fils de John Brown et de Beatrice Jones. Une sorte de jeu dont on peut se retirer à volonté. (p. 85-86)

À la lumière d'une relecture qui installe Stevens èn position d' «auteur» des Fous de Bassan, ce passage évoquerait clairement le pouvoir anonyme d'organisation, de disposition et de témoignage que lui procure la confession par le truchement d'une écriture «fictive». Les souvenirs, les images et les témoignages des Fous de Bassan relèveraient donc en fait de cette voix «autre» qui n'est que le masque du je-scripteur. Ainsi s'expliquerait, par exemple, le fait que les seules instances d' «écriture» motivées par la fiction sont les lettres de Stevens: les autres narrations, caractérisées par Gould comme les manuscrits et les lettres du révérend Jones, de Stevens Brown, et du petit frère arriéré de Stevens, Perceval ${ }^{31}$, sont explicitement des espèces de monologue intérieur et ne peuvent aucunement se lire comme les écrits des personnages. (Même le livre de Nicolas Jones, que l'on imagine être un journal ou des mémoires, met en scène un vieillard somnolant et rêvant dans son fauteuil - et non pas un scripteur en train de rédiger un texte.) Le seul scripteur mis en scène dans le texte, finalement, c'est Stevens lui-même. Les «livres», qui apparentent la structure du texte à l'écriture biblique, et par conséquent suggèrent l'autorité et l'origine douteuse des livres apocryphes, proposent donc une subtile ambiguïté entre le sujet et l'objet de l'écriture.

Cette lecture, nécessairement rétrospective, déplace l'interprétation narrative et féministe du texte. Imaginer Stevens comme «auteur» des Fous de Bassan prêterait une autre dimension à sa structure anaphorique et aux récurrences de toutes sortes. Et, par cette lecture, l'anomalie des voix narratives et des scripteurs qui sont, selon toute évidence, muets, s'éclairerait.

L'étonnante conformité entre la vision du monde proposée par Stevens et celle des autres personnages, surtout des femmes, découlerait du fait qu'il n'y a toujours qu'une seule vision. Pourtant, le déplacement du point de vue et sa

31 Karen Gould, loc. cit., p. 934. 
concentration dans la psychologie de Stevens n'infirmerait pas, en fin de compte, les conclusions féministes qui insistent sur la subjugation des voix féminines et leur émergence dans les forces naturelles. Mais cette vision du monde prendrait une nouvelle signification si elle était perçue comme la reconstruction masculine d'une aliénation vécue comme expérience masculine. La hantise de Stevens et son obsession, c'est bien la femme comme force primordiale et menaçante. Mais proposer cette obsession comme étant celle d'une intentionnalité auctoriale, en l'occurrence hébertienne, c'est peut-être lui attribuer à tort une interprétation du monde qui n'appartient qu'à l'imagination détraquée d'un fou misogyne.

Si le texte représente la tentative de Stevens d'organiser ses souvenirs, alors le pouvoir que détiennent les femmes n'est que celui que leur confère son obsession maladive, et ce pouvoir est considérable. C'est ainsi que la «voix des femmes» ne quitte jamais le cercle du mythe primordial où Stevens, l'historien de Griffin Creek, les enferme. Si Nora est l'«Ève nouvelle», c'est que Stevens le veut ainsi, jusqu'à inscrire sa destinée à lui et celle de tout le monde dans un cercle vicieux de désir, de tentation, de chute et de perte mutuelle.

Parmi d'autres énigmes narratives qui ont attiré l'attention des critiques, les non-dits dans le texte, l'omniscience et la prescience des personnages s'expliqueraient également par le déplacement du point de vue narratif vers Stevens. Dans le discours de Stevens, la prescience prend la forme d'ellipses qui seraient autant d'indices, selon Kathryn Slott, du refoulement du désir coupable, innommable ${ }^{32}$. Et Neil Bishop décrit le même phénomène comme étant des prolepses irréalistes et invraisemblables dans des lettres datées d'avant le 31 août 1936, autant d'indications que l'écriture des Fous de Bassan a des buts tout autres que le réalisme, notamment le suspense, la tension et l'intérêt romanesque ${ }^{33}$. Mais certaines phrases: $N e$ pas laisser la suite de mon histoire à Griffin Creek se dérouler jusqu'au bout (p. 80) et Il faut pourtant que je te dise la suite (p. 82) impliqueraient non seulement la conscience chez Stevens de jouer un rôle dans une histoire, mais aussi la connaissance que cette histoire a une suite néfaste, et donc une intrigue. Et c'est surtout dans le récit de Nora que cette prescience échappe à toute explication narrative. Même en tenant compte d'une certaine fluidité temporelle dans son discours, il faut, selon la logique du récit, priver ce personnage d'une omniscience au sujet de son avenir qui ne soit pas simple conjecture. Or, comment expliquer le présage d'un malheur qui impliquera tous les personnages du drame futur dans un avenir où ils seront liés par le sang et la mort?

Stevens, debout, inondé de lumière, l'ombre noire de son chapeau sur ses yeux, vient de faire son apparition dans l'encadrement de la porte. $\grave{A}$ partir de là tout va aller très vite à Griffin Creek. Mon oncle Nicolas, ma tante Irène, Stevens, Perceval, Olivia et moi serons tous

32 Kathryn Slott, «Repression...», loc. cit., p. 298-299.

33 Neil Bishop, «Distance...», loc. cit., p. 117. 
emportés par le mouvement de notre propre sang, lâché dans la campagne, au grand galop de la vie et de la mort. (p. 121)

Les sens produits par les énigmes narratives «invraisemblables» ne sauraient s'épuiser par une explication qui valorise leur fonction uniquement en terme de but esthétique anti-réaliste. En tant qu'indices textuels, ces signes narratifs sont générateurs de sens qui transcendent le niveau des effets de style. Leur réintégration dans une logique signifiante bouleverse la lecture qui tend vers le fragile rêve de multiples identités narratives uniques et transparentes à elles-mêmes.

\section{Clôture}

Revenons à notre point de départ, la question de l'incertitude qui plane toujours sur la résolution du crime. Si Stevens est le seul auteur d'une confession où il raconte sa version du drame, les multiples points de vue n'existent pas. Stevens aurait organisé les «faits» en attribuant à ses personnages les motivations qu'il lui plaît d'imaginer. Or, Perceval - cet autre lui-même reste une énigme. De toute évidence, le texte nous le présente comme détenteur d'un savoir privilégié qu'il ne veut pas divulguer.

Au niveau de tous les récits, il y a une certaine généralisation de la culpabilité qui touche même les deux filles. Cette généralisation s'expliquerait par le fait que Stevens, tout en se confessant, s'exempte de la seule responsabilité en montrant la profondeur de la corruption et de la dégradation qui n'avaient pour source ni un individu ni un moment historique, mais qui existaient et évoluaient depuis les origines. Or, le seul personnage qui soit clairement exonéré de cette culpabilité générale est Perceval, dont l'innocence est absolue en raison de son enfance éternelle. Il ne possède pas la possibilité de déchoir. Toutefois, les contradictions proliferent. Privé de langage, Perceval est néanmoins doté d'une certaine faculté de comprendre et d'une connaissance supérieure des faits, de sorte que la plupart des indices de l'enquête qui apparentent les Fous de Bassan au roman policier sont fournis dans son récit. Et pourtant, il est clair que Perceval lui-même continue à croire à l'innocence de Stevens. Répétant une phrase clé, Non, non, ce n'est pas Stevens, phrase qui résonne ailleurs dans le livre comme un avis au lecteur, Perceval insiste jusqu'à la fin sur une vérité insolite.

Et au sujet des «faits» établis autour du soir du 31 août, le texte laisse planer un doute considérable. Prenons, par exemple, la présence possible des personnages sur la plage ce soir-là. Selon Stevens, tous les hommes auraient été présents: Perceval, que la lune excite (p. 106); Nicolas, qui erre souvent sur la grève, de jour comme de nuit (p. 107) depuis la mort de sa femme, et Stevens. Or, dans son témoignage à l'inspecteur, rapporté par Perceval, Stevens (ou bien Perceval) supprime le nom de Perceval tout en constatant la présence du pasteur que celui-ci confirme.

Ainsi, la présence ou l'absence de Perceval sur la plage devient le chaînon manquant pour l'établissement des «faits». Perceval affirme: Mes parents jurent qu'ils m'ont enfermé dans ma chambre à huit heures précises, le soir du 31 août. Comme d' habitude. (p. 163) Mais les mêmes parents jurent aussi que 
Stevens est rentré vers neuf heures (p. 159), alors que le crime aurait été commis vers neuf heures trente. Et Perceval lui-même ne fournit aucune évidence de la véracité ni de la fiabilité de son témoignage. Il insiste au contraire sur le fait qu'il ne dira rien, et les indices qu'il tait ne semblent pas tous jouir d'un même statut d'importance: Je ne dirai rien. Ni le petit couteau. Ni le gros et le petit bateau. Ni l'auto étrangère. Ni le chapeau perdu de Stevens [...], tout ça que je cache dans ma tête et qui me gêne pour dormir. (p. 158)

Dans la reconstruction des éléments significatifs pour la résolution du crime, seuls le petit bateau de Stevens et son chapeau perdu semblent pertinents. Si, d'une part, Perceval accorde un statut égal à tout ce qu'il a vu, il ne fait aucune mention, d'autre part, de la présence de Stevens sur la plage, qu'il aurait vu de sa fenêtre ce soir-là. Si Perceval a une connaissance supérieure des faits, il la tait non seulement devant les personnages du drame, mais également devant le lecteur.

L'ambiguiité de Perceval comme narrateur témoin s'accroît par l'effet de la dernière lettre de Stevens et est centrée sur deux problèmes principaux. Premièrement, en avouant qu'il n'a jamais aimé personne sauf Perceval, cet autre luimême, Stevens prie pour que cet innocent l'accueille en Paradis. Que Perceval aille au Paradis s'accorde bien avec son innocence enfantine. Mais qu'est-ce qui justifie la croyance chez Stevens, qui selon son propre témoignage avait fait trop de lectures bibliques (p. 89) dans son enfance, que lui, meurtrier impuni, se rendra au Paradis? Par le truchement du dédoublement entre Perceval et Stevens, il semble s'opérer un échange d'attributs. Si la folie de Perceval extériorise 1'alter ego submergé et refoulé de Stevens, Perceval ne serait-il pas aussi représentatif de l'innocence sauvage et déchaînée de Stevens? Si l'on prend au pied de la lettre l'identification continue entre Stevens et son frère, les deux personnages finissent par n'en faire qu'un seul. Stevens et Perceval, tout comme Pam et Pat, Nora et Olivia, forment des jumeaux indissociables. Selon ce raisonnement, Perceval partagerait la culpabilité de Stevens, tout comme Stevens partagerait l'innocence de son frère, et c'est cette innocence qui est mise en valeur dans la dernière prière de Stevens.

Le deuxième problème, guère plus soluble, est celui posé par la question de l'extorsion des aveux. Le motif de l'extorsion est clair: McKenna empoche une récompense pour la capture du criminel. Mais la question se pose: quelle pression McKenna aurait-il exercée sur Stevens pour lui faire avouer qu'il est assassin? À prime abord, l'explication se trouverait dans la violence exercée contre Stevens, selon le témoignage de Perceval, lors de l'interrogatoire. Mais cette explication ne semble pas satisfaisante. Malgré le cou cassé (p. 192), rapporté par Perceval, la violence perpétrée par l'inquisiteur serait plutôt morale:

Ça ne ressemble pas à mon frère Stevens. Sans regard. Ni fierté. Les traits tirés. Les jambes et les bras trop longs.

[...]

Mon frère Stevens retourne dans la chambre, l'air d'un chien qu'on tire par sa laisse et qui se résigne mal à obéir. La voix de McKenna se fait implorante, douce, presque tendre. 
- Tu comprends, il faut absolument tirer ça au clair. Je suis venu pour ça.

Il répète, rassurant et jovial.

- Tu te sentiras mieux après. (p. 191-192)

Quant à l'extorsion des aveux, Stevens affirme dans sa dernière lettre (p. 243) que sa confession est finale et - on le présuppose pour ce qui est du récit du meurtre - véridique. Mais, du point de vue de Stevens, qu'est-ce qui provoquerait un aveu forcé, que celui-ci soit vrai ou faux? L'information que nous tenons à son sujet ne justifierait pas l'efficacité de la force, ni physique ni morale. Le rôle de Perceval dans l'histoire, comme témoin et double de Stevens, devient de plus en plus problématique. En racontant dans sa dernière lettre le progrès dans les recherches des vêtements des victimes, Stevens a recours à une ellipse qui s'explique mal dans le cadre d'une confession totale et finale:

Autant chercher une aiguille dans un tas de foin. Bien fin qui trouvera. Le running shoe de Nora plein de sable, l'air d' un coquillage enfoui. C'est mon frère Perceval qui... Ne peut que crier et pleurer. C'est fou d'ailleurs ce que cet enfant m'est attaché. Ne me trahira jamais. (p. 243)

Si l'aveu final de Stevens semble garantir sa culpabilité, c'est qu'à cette confession est accordée une crédibilité plus grande que ne le sont d'autres parties de cette lettre. Dans la lecture critique, la clôture apparente fournie par la confession l'emporte sur d'autres indices textuels contradictoires: la protestation que sa folie ne serait que le résultat de la guerre, par exemple; et l'aveu que sa hantise du meurtre ne serait que l'effet d'un rêve récurrent: Si je persiste à voir les petites Atkins, foudroyées à mes pieds sur le sable et moi debout, audessus d'elles, tout bête et creux, vide jusqu'à la moelle de mes os, c'est que je n'en finis pas de rêver. (p. 241) En fait la confession ne dissipe aucunement l'accumulation d'énigmes qui atteint son apogée dans la demière lettre de Stevens. Si, en effet, un doute plane sur la résolution du mystère, ce doute est inscrit au niveau du discours et de la structure romanesques, dans l'ambiguïté de la narration et dans l'universalité des forces maléfiques qui circulaient à Griffin Creek pendant l'été 1936.

La demière confession de Stevens Brown est conforme aux conventions du roman policier par la place privilégiée qu'elle occupe. En épilogue à la «vraie action», elle tend vers une fin et une finalité qui projettent la résolution nette d'une énigme par l'épuisement du sens textuel, suscitant ainsi les présupposés de clôture, d'unité et de vérité. Les stratégies mises en place sur le plan du point de vue et de la construction des savoirs narratifs infirment toutefois ce modèle.

Or, un paradoxe inhérent à notre lecture nous aurait-il échappé? En voulant lire les Fous de Bassan comme «le livre de Stevens Brown», nous aurions effectué une réduction de l'ouverture qui pourrait résulter d'une lecture plurivoque de la narration. Nous suggérons, en revanche, une remise en question du présupposé de l'autorité narrative qui mine la logique de la vérité du sujet 
inscrite dans le modèle des voix narratives multiples. C'est ainsi que, se masquant derrière le faux présupposé d'un sujet du discours transparent à luimême, la représentation du sujet dans le discours résulte en fait d'un travail par l'autre. Dans ce sens, Stevens, le seul personnage à être à la fois sujet et objet de son propre discours, se crée au même titre qu'il crée les autres «personnages» de son récit. Bloquée au niveau de sa médiation discursive par Stevens, la (fausse) transparence proposée entre le discours et le sujet assume une opacité qui empêche le retour à une vérité originelle.

Ce relevé des problèmes de logique et d'interprétation posés par les Fous de Bassan ne prétend pas à l'exhaustivité. Tout au plus espérons-nous avoir indiqué un réseau d'énigmes qui constitue un résidu problématique laissé de côté par les lectures antérieures. Ainsi, nous suggérons que le pouvoir du Verbe dans ce texte équivoque se déplace et s'esquive en même temps que la vérité: que le Verbe et la Vérité sont incommensurables, que le tout n'est pas dit. 